\title{
COMPARISON OF DEFUZZIFICATION METHODS IN THE CASE OF AIR CONDITIONING SYSTEMS
}

\author{
Sinan KOÇAK ${ }^{1}$, László POKORÁDI ${ }^{2}$ \\ ${ }^{1}$ Óbuda University, Doctoral School on Safety and Security Sciences, Budapest, Hungary \\ sinan.kocak@bgk.uni-obuda.hu \\ ${ }^{2}$ Óbuda University, Institute of Mechatronics and Vehicle Engineering, Budapest, Hungary \\ pokoradi.laszlo@bgk.uni-obuda.hu
}

\begin{abstract}
This paper provides the design for air conditioning system and two different defuzzification methods. The air conditioner intelligent system is the control of two control parameter: the temperature and the humidity of the area. The inputs received by the temperature and humidity sensors give the closest output values for the air conditioning system. The simulation result shows temperature and humidity of the heat exchange by using defuzzification methods of centroid and middle of maxima (MoM) in the Fuzzy Logic Toolbox of MATLAB package program.
\end{abstract}

Keywords: Fuzzy logic, defuzzification methods, air condition.

\section{Introduction}

Fuzzy logic is a branch of algebra that is based on thinking like human beings and transforming this thinking into mathematical functions. The greatest feature of fuzzy logic is mathematical discipline based on Fuzzy set theory instead of binary Aristotle logic (yes or no, 1 or 0, like or not like etc.). However, fuzzy logic takes values between those two binary values: it operates using intermediate values $(0.6$ - 0.1$)$ instead of $0-1$ values for few, many, little, normal, medium, long.

The concept of fuzzy logic was first introduced by the Azerbaijani Scientist L. A. Zadeh in middle of the 1960s as a mathematical study. This theory is used in areas where mathematical expressions are difficult to obtain [1]. The first fuzzy logic control application was implemented by Mamdani in 1974 with the control of the steam machine. It was shown that Zadeh's approach was easily handled by the computer [2].

The process of converting the fuzzy output to a crisp value is said to be defuzzification. The defuzzification process enters the circuit as the last element of the fuzzy processor of the air conditioning system. The rule results obtained in the fuzzy rule processing unit are evaluated in the optimizer and converted to a definite result.

In this study, air conditioning system modelling will be examined in order to provide a good example system for the basic principles of fuzzy logic control systems and showing two different defuzzification methods. Examination of small temperature variations in the time domain system that continuously affect the system output using fuzzy logic control systems is of great importance.

\section{Fuzzy Knowledge-Based Control}

Fuzzy knowledge-based (FKBC) control is a method of developing a control algorithm that has been widely used in recent years. It is not appropriate to apply traditional control methods, especially in complex systems. Applying these methods in such situations is both expensive and presents difficulties. From this point of view, complicated systems can be easily modelled by fuzzy logic. In many complex applications, many academic studies and applications have achieved high performance with fuzzy logic. However, fuzzy logic is directly related to experience. Correct rule bases and curriculum identification en- 
sure that results that are closest to actual results are obtained, depending on experience. This experience can take a lot of time depending on the application. This situation should be seen as a disadvantage of fuzzy logic.

The principal design parameters of an FKBC consist of scaling factors, fuzzification, rule base, and defuzzification methods, etc.

\section{Air conditioner Controlling Process with Fuzzy Logic}

An air conditioning installation needs very large control. Because the temperature and humidity control parameters interact with each other. That means the room temperature automatically changes when the humidity changes, even if it is a requested value. Temperature and relative humidity RH (\%) of regulators have to be associated with each other.

The aim of air conditioning systems is the control of temperature and humidity at the same time. The room temperature should be between 18 and $24^{\circ} \mathrm{C}$ depending on humidity. The optimal relative humidity is between 35 and $70 \%$. Usually, it can be achieved by controlling room temperature in relation to the inside air. In addition, people feel higher temperature is more comfortable with a lower humidity than with a higher humidity.

Fuzzy logic is the favourable approach for air conditioning systems. The magnitude of settings is practically incompatible, it can be converted to the direct concept in the linguistic term. People are not fixed to specific temperature and humidity. Conversely, humans accept large areas as being comfortable. Consequently, this comfort zone (Figure 1.) is the result of a person feeling relaxed in a room where the temperature is low and the humidity slightly higher.

The working principle is the same as in an air/ air heat pump with a hot- and a cold side. When the heat is flowing from inside to outside, the compressor and two fans are powered by the current. The transport medium is the refrigerant which evaporates at temperatures below room temperature in the inside unit. Heat energy is needed to evaporate the refrigerant until all liquid inside the evaporator is converted into steam. A compressor will raise the pressure and with it the temperature of the steamed refrigerant higher than the ambient temperature. At this point, the stored heat can be automatically removed to the outside by condensing the refrigerant back into

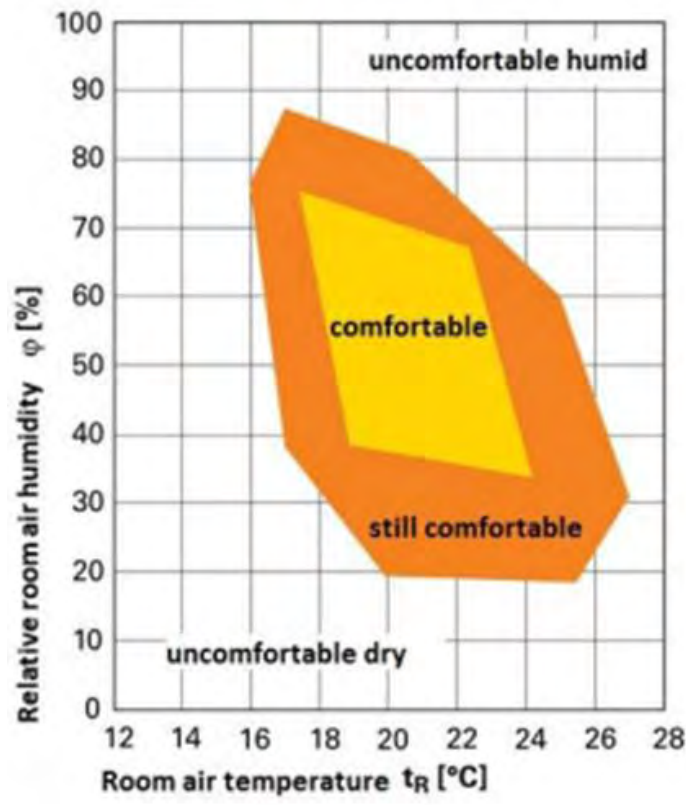

Figure 1. Comfort zone as a function of temperature and humidity (source: [3])

liquid. An expansion valve releases pressure and with it the temperature at the indoor unit back to the starting point. Fans on both sides support the heat transfer. The advantage of using this cycle is that more cooling capacity can be provided as needed.

\section{Case Study}

Fuzzification is the process of transforming input variables and output variables into linguistic expressions. For this reason, the initial input and output variables are determined as input variables for the air conditioning system; in the case of internal temperature, the effect of temperature and humidity was selected and an output variable; Heater and humidifier. Linguistic expressions are defined by the degree of input and output variable membership. The membership grades of output variables are also adjusted according to the weights of the rules. In the this study, the centroid and middle of maxima methods are used to measure the variables.

It is possible to make the system more sensitive by reducing the areas covered by the specified linguistic expressions of inside temperature and other variables, or by adding new qualifiers. The alternatives here depend on the choice of the person to implement. 


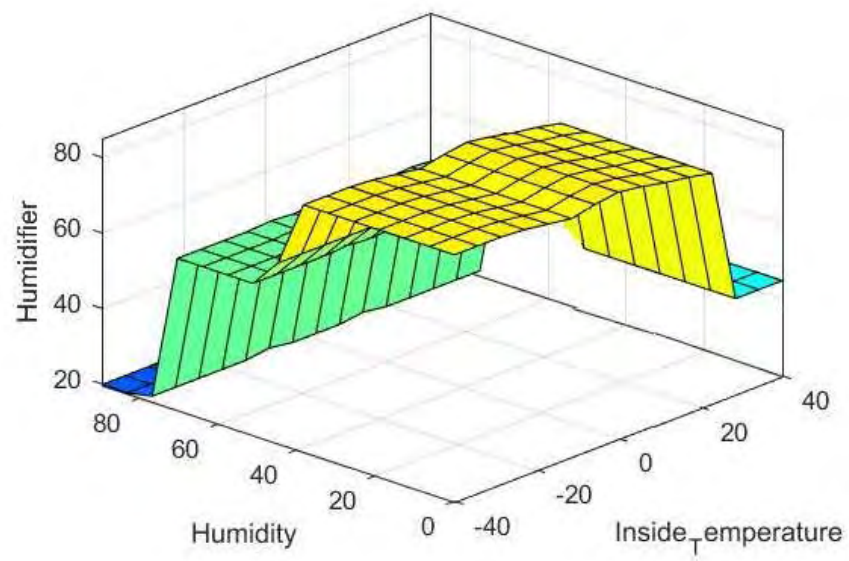

Figure 2. Defuzzification Surface of Humidifier in case of Mom method

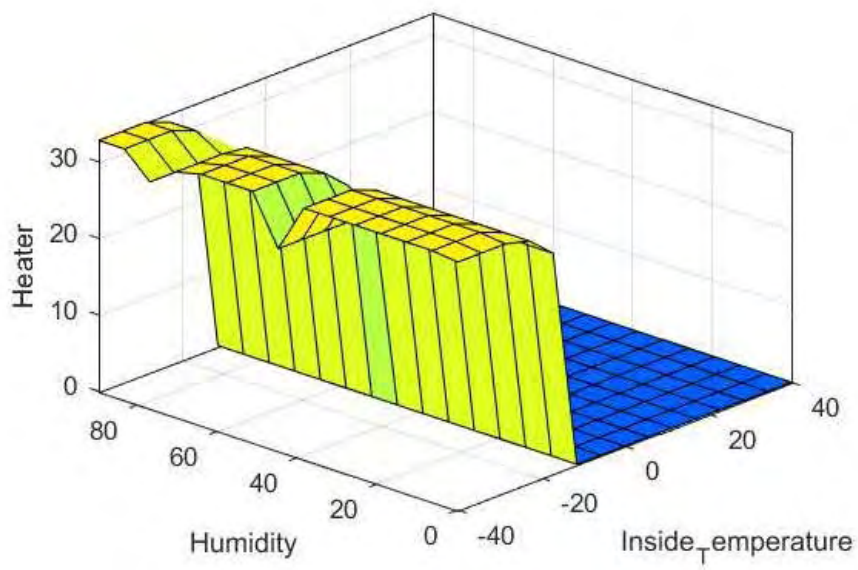

Figure 3. Defuzzification Surface of Heater in case of Mom method

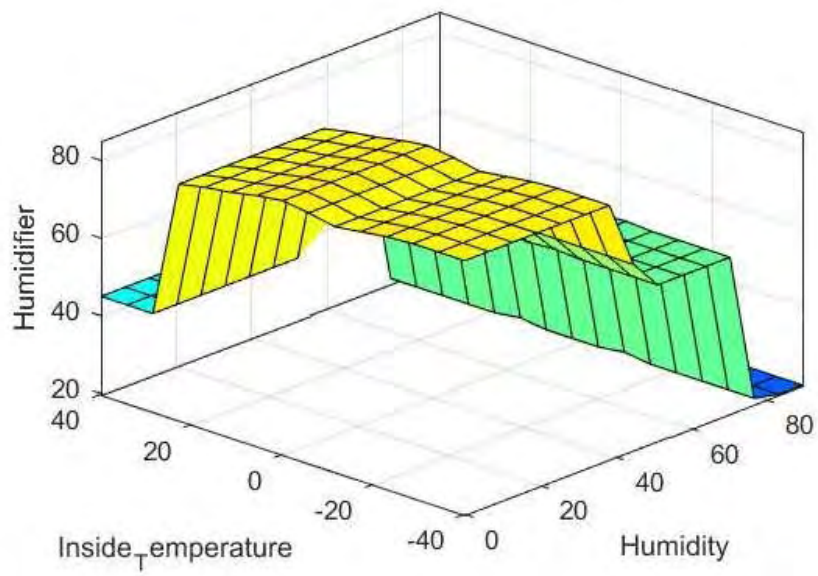

Figure 4. Defuzzification Surface of Humidifier in case of Centroid method 


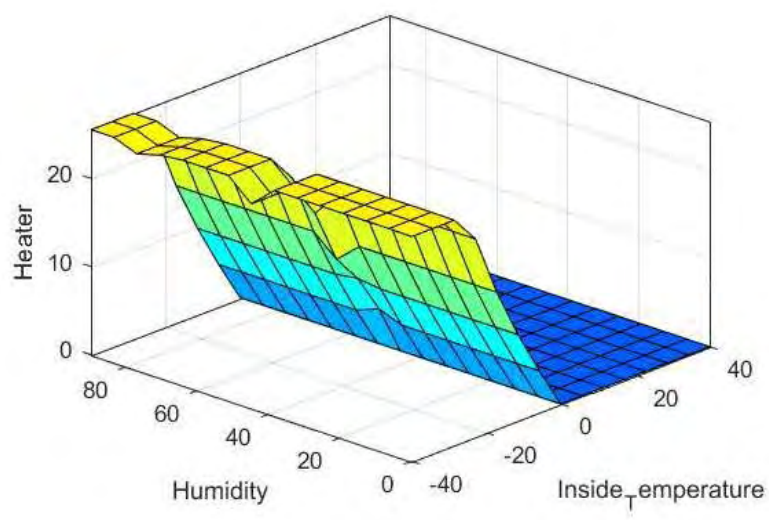

Figure 5. Defuzzification Surface of Heater in case of Centroid method

Linguistic expressions used for fuzzification, the inside temperature; cold, normal and hot. Membership functions here are at the discretion of the practitioner. Different values can be given according to the area where the system is used and linguistic expressions can be increased. For example, different linguistic qualifiers and values for a system to be applied only for the temperature control of electronic devices, different linguistic qualifiers and values can be created for a system to be designed for human health (Figure 2-5.).

The major problems that arise in the design of control systems are that it is difficult and complex to construct the mathematical model of the system to be controlled. It has been observed that the temperature and humidity of the heat exchange accordingly observed to be softer and the mechanisms for changing the desired humidity value has enabled us to get faster.

\section{Summary}

In this study, controlling the design of the air condition system with Fuzzy Logic was performed and a simulation was prepared in the Fuzzy Logic Toolbox of MATLAB package program to observe the results related to the system.

\section{References}

[1] Zadeh L. A.: Fuzzy sets. In Fuzzy Sets, Fuzzy Logic and Fuzzy Systems: Selected Papers by Lotfi A Zadeh (Eds. George J Klir, Bo Yuan) Word Scientific, 1996, 394-432.

https://doi.org/10.1142/9789814261302_0021

[2] Mamdani E. H.: Application of fuzzy algorithms for control of simple dynamic plant. Proceedings of the Institution of Electrical Engineers 121/12. (1974) 1585-1588. https://doi.org/10.1049/piee.1974.0328

[3] Kosonen R., Tan F.: Assessment of productivity loss in air-conditioned buildings using PMV index. Energy and Buildings 36/10. (2004) 987-994. https://doi.org/10.1016/j.enbuild.2004.06.021 